\title{
REAL-TIME DIAGNOSTICS FOR ROS RUNNING SYSTEMS BASED ON PROBABILISTIC PATTERNS IDENTIFICATION
}

\author{
S. Vechet, J. Krejsa*
}

\begin{abstract}
Autonomous mobile robots consists of various software modules to achieve given goal, including solving complex navigation tasks as localization, mapping or path planning. These tasks are highly dependent on the quality of data measured and gathered from hardware subsystems. Using Robot Operating System (ROS) as integration basis reduces the development effort and time to market. While ROS framework itself is considered as reliable and stable to run even soft real-time tasks, in case of any internal failures on data misreadings can be problematic to debug or even identify the problem for common user. Due to this unpleasant situations we develop a virtual assistant, internally represented as diagnostic expert system, to help users to identify and possibly fix the problem.
\end{abstract}

Keywords: Diagnostic Expert System, Bayesian Networks, System Diagnostic, Robot Operating System, ROS.

\section{Introduction}

The Robot Operating System (ROS) is a framework originally developed for DARPA Grand Challenge competition which took a place in Mojave Desert during summer of 2014 where a group of autonomous cars should travel a 150 miles long path without any human operator assistance.

The ROS takes care about inter-process communication based on publisher-subscriber architecture, it handles data logging, visualization, path planning (Masek (2015)), navigation (Krejsa (2010)) and partial in system diagnostics.

The main principle is, that every activity which needs to be executed is implemented as separate node and can be published (publish some internal information to the other nodes in the system), subscriber (it only listens what other nodes publish) or both. The user don't need to care if the nodes are executed on local machine or in shared network, which simplify the system design, where multiple independent PC can be used without limitation. Typical network of nodes can be seen on figure 1. It represents the communication network on robot BREACH (see Krejsa (2018); Hrbacek (2010)).

\section{Problem definition}

The internal tools of ROS helps developers to debug the system while development process however they are very hard to use for common user, moreover for common customer. Standard diagnostic tools are:

- rostopic - command line tool for topics diagnostics,

- RQT Graph - shows the communication network (also presented on figure 1.),

- R-Viz - main graphics user interface for visualisation of the all information within the system, partially usable as a graphics user interface for customers.

Assoc. Prof. Stanislav Vechet, Ph.D., Assoc. Prof. Jiri Krejsa, Ph.D.: Institute of Thermomechanics AS CR, v.v.i., Technicka 2, 616 69, Brno, Czech Republic, vechet.s@fme.vutbr.cz 


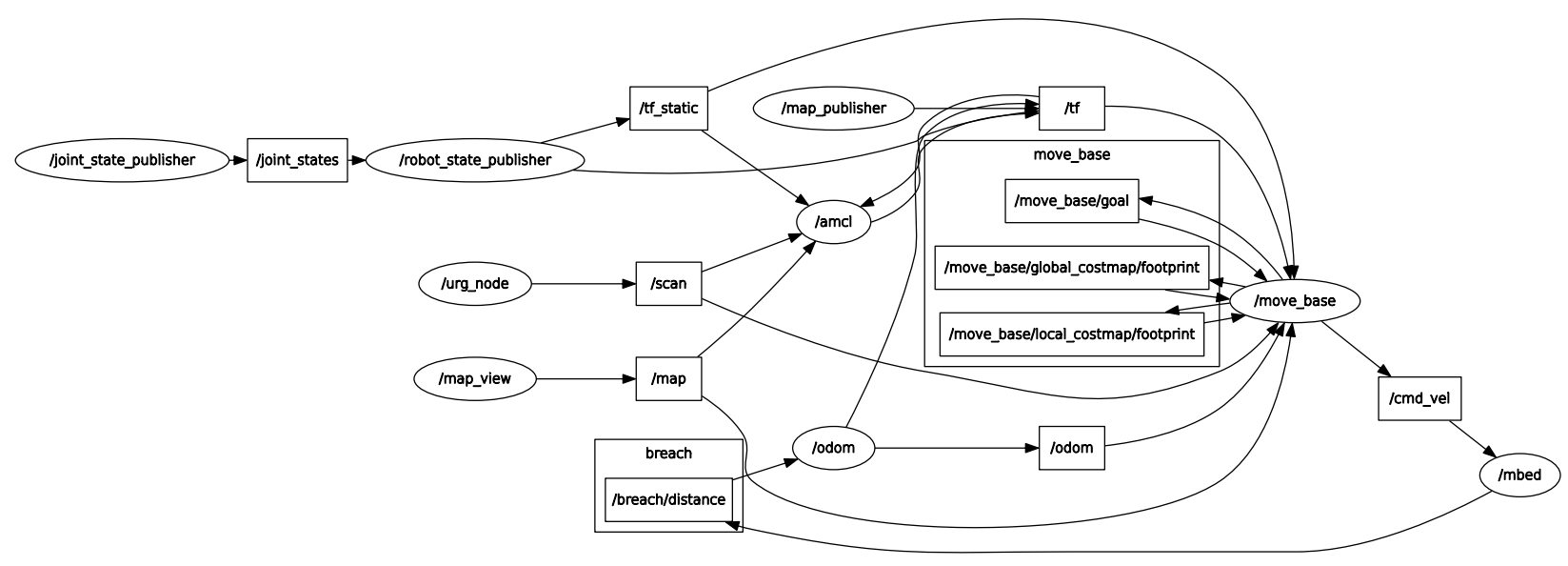

Fig. 1: ROS nodes example architecture

For description of the above mentioned tools and how to use it please refer ROS (2019).

Our goal is to prepare diagnostic system which doesn't requires an highly skilled or experienced user to diagnose where the problem is and find a way how to solve it.

The basic principle is to monitor the data flow within the system and detect an anomalies in the time domain. The anomaly detection is done via using the probabilistic approach where a patterns in received data are searched. If the pattern changes in time it causes an anomalous behaviour of the system.

The method principle is based on observation that the common problem is connection between processes or overload of particular subsystems which causes the data loss. The data loss is responsible for loss off accuracy or system failure (Krejsa (2014)).

We design an approach which detects the time differences between data received from each node and constructs a probabilistic pattern (Vechet (2016)) which can be observed in the data flow. If the pattern changes in time the system needs to decide Merritt (1989) whether is the standard behaviour or possible pathological behaviour which causes the system failure in the future.

\section{Probabilistic approach}

The main idea is to use Bayesian probability Korb (2010) to calculate the Believe that the system is operational based on time differences in received data.

$$
P(O k \mid 1, d t)=\eta^{-1} P(1 \mid O k) P(d t \mid O k) P(O k)
$$

where

$$
\eta^{-1}=P(1 \mid O k) P(d t \mid O k) P(O k)+P(1 \mid E r r) P(d t \mid E r r) P(E r r)
$$

and

- $d t$ - time difference between two consequently received data,

- 1 - denotes the event that some data was received,

- $O k$ - denotes the system is normal shape,

- Err - denotes the system is in erroneous state, 
- $P(O k \mid 1, d t)$ - believe that the system is operational,

- $P(O k)$ - system reliability,

- $P(1 \mid O k)$ - predicted probability that some measurement will take a place,

- $P(d t \mid O k)$ - probability calculated as a mean of time differences from received data,

- $P(1 \mid E r r)=1-P(1 \mid O k)$,

- $P(d t \mid E r)=1-P(d t \mid O k)$.

\section{Experimental results}

Experimental results where measured on mobile platform BREACH. We monitor the communication in the system which is represented on figure 2 . by the measurements series.

Where the values -1 means nothing received and values 1 means data received. The series named probability shows the probability $P(1 \mid O k)$ - that gives an estimate that some data will be observed.

The figure 3. shows the believe that the observed node is operational, there can be seen a drop in believe when the data stops to be received from the publisher and the probability is going down to zero. This means that the node is in erroneous shape and consequent diagnostic actions need to be executed.

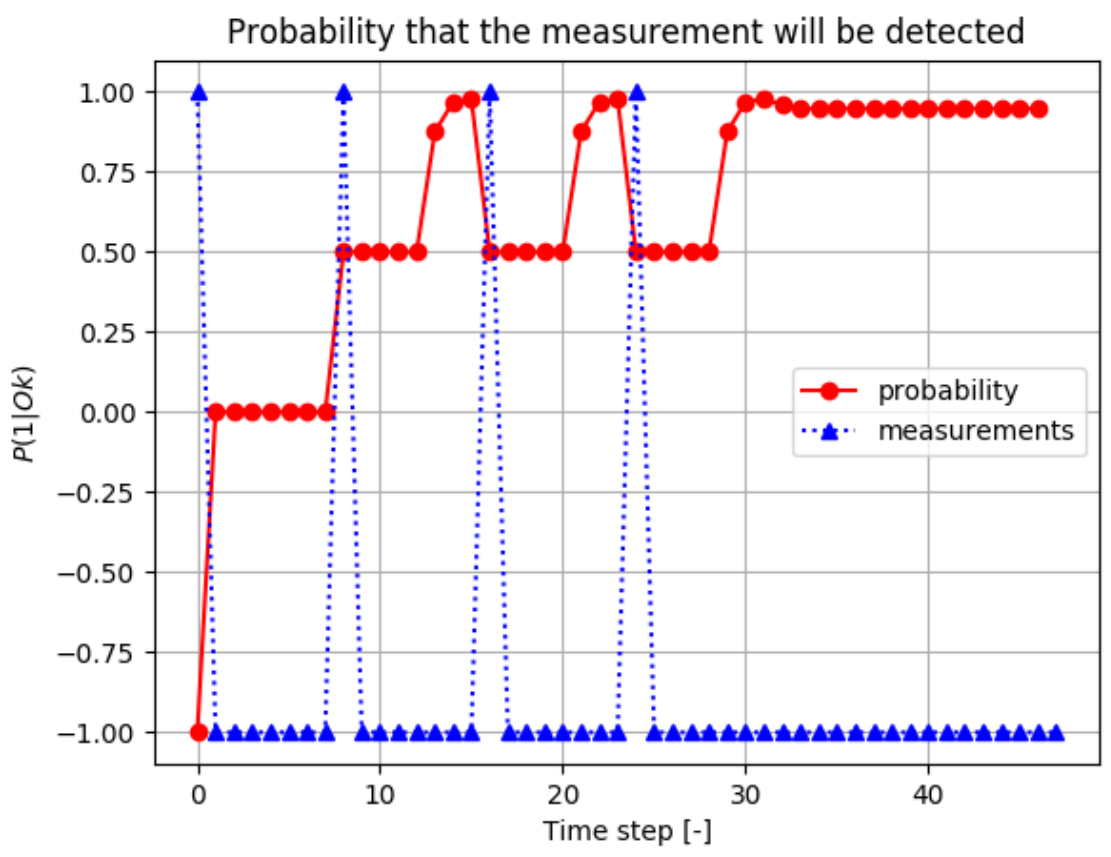

Fig. 2: Probability that the measurement will be detected

\section{Conclusions}

We present a first step to independent system diagnostic where patterns in data flow are searched. The patterns detection is based on time difference in received data and the probability that some observation should be detected is calculated. When a probabilistic pattern changes in time the probability of an undesirable behaviour is calculated and shown to the user. This approach seems to be favourable due to the fact that no pre-training is necessary (e.g. no need to learn what good or erroneous behaviour is). The diagnostic can be running on the fly while the system is in both the operational or development stage.

The future goal is to use the believe that the system is operational to feed the high level decision networks to search of the most probable cause of the problem and find appropriate solution. 


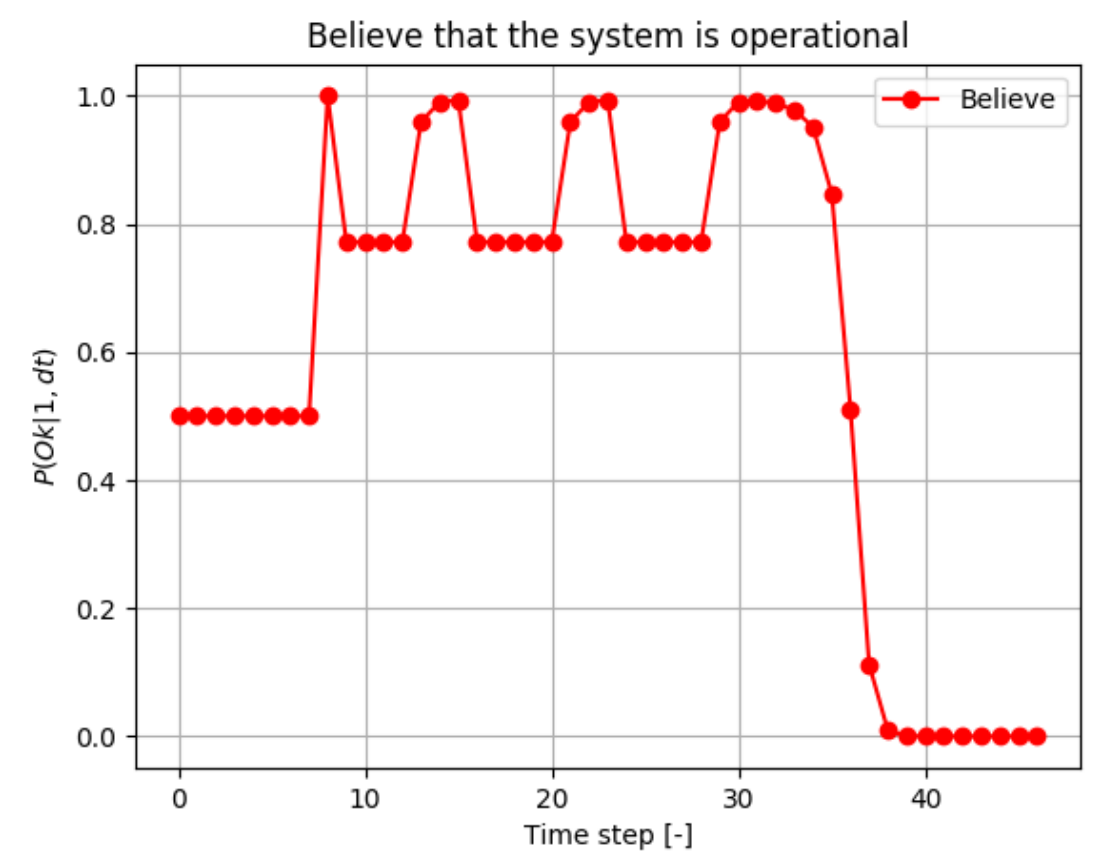

Fig. 3: Believe that the system is operational

\section{Acknowledgement}

The results were obtained with institutional support RVO 61388998 of the Institute of Thermomechanics AS CR v.v.i.

\section{References}

Hrbacek, J., Ripel, T., Krejsa, J. (2010) Ackermann mobile robot chassis with independent rear wheel drives. Proceedings of 14th International Power Electronics and Motion Control Conference (Epe-Pemc 2010). Doi 10.1109/Epepemc.2010.5606853. WOS:000319521600268

Korb, K. B. and Nicholson, A. E. (2010) Bayesian Artificial Intelligence, Second Edition, CRC Press, Inc., Boca Raton, FL, USA, 2010, ISBN 1-58488-387-1.

Krejsa J., Vechet S., Chen K. (2014), Multiple Indoor Robot Localization using Infrared Beacons, Engineering Mechanics 2014, pp. 336-339, WOS: 000364573900077

Krejsa, J., Vechet, S. (2010) Odometry-free mobile robot localization using bearing only beacons. Proceedings of 14th International Power Electronics and Motion Control Conference (Epe-Pemc 2010). Doi 10.1109/Epepemc.2010.5606893. WOS:000319521600308

Krejsa J. and Vechet S. (2018) Fusion of Local and Global Sensory Information in Mobile Robot Outdoor Localization Task. In: Proceedings of the 2018 18th International Conference on Mechatronics - Mechatronika (ME) 2018. Brno, pp 296-300.

Mašek, P.; Růžička, M. (2015) A Task Planner for Autonomous Mobile Robot Based on Semantic Network. In Advanced Mechatronics Solutions. Advances in Intelligent Systems and Computing. Switzerland: Springer International Publishing, 2015. p. 637-642. ISBN: 978-3-319-23921- 7. ISSN: 2194-5357.

Merritt, D. (1989) Building experts systems in Prolog. Springer-Verlag, New York (1989) ISBN:978-1-4613-8913-2

Robot Operating System, available online at www.ros.org, retrieved 2019

Věchet, S., Hrbáček, J., Krejsa, J. (2016) Environmental Data Analysis for Learning Behavioral Patterns in Smart Homes. In Proceedings of the, 2016 17th International Conference on Mechatronics-Mechatronika (ME) 2016. 1. Prague: Czech Technical University in Prague, 2016. p. 386-391. ISBN: 978-80-01-05882- 4. 